\title{
Range of the Fractional Weak Discrepancy Function
}

\author{
Alan Shuchat \\ Department of Mathematics \\ Wellesley College \\ Wellesley, MA 02481 USA \\ Randy Shull \\ Department of Computer Science \\ Wellesley College \\ Wellesley, MA 02481 USA \\ Ann N. Trenk \\ Department of Mathematics \\ Wellesley College \\ Wellesley, MA 02481 USA
}

February 21, 2006

\begin{abstract}
In this paper we describe the range of values that can be taken by the fractional weak discrepancy of a poset and characterize semiorders in terms of these values. In [6], we defined the fractional weak discrepancy $w d_{F}(P)$ of a poset $P=(V, \prec)$ to be the minimum nonnegative $k$ for which there exists a function $f: V \rightarrow \mathbf{R}$ satisfying (1) if $a \prec b$ then $f(a)+1 \leq f(b)$ and (2) if $a \| b$ then $|f(a)-f(b)| \leq k$. This notion builds on previous work on weak discrepancy in $[3,7,8]$. We prove here that the range of values of the function $w d_{F}$ is the set of rational numbers that are either at least one or equal to $\frac{r}{r+1}$ for some nonnegative integer $r$. Moreover, $P$ is a semiorder if and only if $w d_{F}(P)<1$, and the range taken over all semiorders is the set of such fractions $\frac{r}{r+1}$.
\end{abstract}




\section{Introduction}

We begin with some preliminaries. Among the posets that appear repeatedly in this paper are those of the form $\mathbf{r}+\mathbf{s}$, which consists of a chain of $r$ elements and a chain of $s$ elements and no additional comparabilities. Familiar classes of posets can be defined in terms of forbidden $\mathbf{r}+\mathbf{s}$ configurations, for example, linear orders (or chains) are posets with no induced $\mathbf{1}+\mathbf{1}$. Weak orders can be defined as posets with no induced $\mathbf{2}+\mathbf{1}$. It is not hard to see that the following definition is equivalent: $P=(V, \prec)$ is a weak order if and only if there is a real-valued function $f: V \rightarrow \mathbf{R}$ so that $a \prec b$ in $P$ if and only if $f(a)<f(b)[1]$. Thus the elements of a weak order can be ranked by a function which respects the ordering $\prec$ and issues a tie in ranking between incomparable elements. Such ranking functions are useful in applications and thus it is desirable to extend the notion of ranking to general posets.

\subsection{Weak Discrepancy}

The initial work on this subject was done in [8] with the definition of $k$-weak orders and an algorithm for computing the minimum $k$ for which a poset is a $k$-weak order. This minimum $k$ is a measure of how far a poset is from being a weak order, and is called the weakness of a poset in [3] and later named the weak discrepancy in [6] and [7].

Definition 1 The (integral) weak discrepancy of a poset $P=(V, \prec)$ (denoted by $w d(P))$ is the least nonnegative integer $k$ for which there exists an integervalued function $f: V \rightarrow \mathbf{Z}$ satisfying

(i) if $a \prec b$ then $f(a)<f(b) \quad$ ("up" constraints)

(ii) if $a \| b$ then $|f(a)-f(b)| \leq k$. ("side" constraints) Such a labeling is called an optimal weak labeling of $P$ (or of $V$ ).

Figure 1 shows the poset $\mathbf{3}+\mathbf{2}$ which has weak discrepancy equal to 2 . An optimal weak labeling is given by $f\left(a_{0}\right)=0, f\left(a_{1}\right)=1, f\left(a_{2}\right)=2, f\left(a_{3}\right)=0$, $f\left(a_{4}\right)=1$.

Weak discrepancy is well-defined since we may use a ranking defined by the height of an element in a linear extension. Definition 1 is motivated by problems such as the following. A manager can partially order the employees in her division based on their value to the company, yet she must assign a salary level to each employee. The "up" constraints ensure that a more valuable employee receives a higher salary. The "side" constraints are fairness conditions that restrict the salary discrepancies between incomparable employees. For a weak order, sets of incomparable employees (antichains) are assigned the same salary level and the weak discrepancy is zero ( $k=0$ satisfies the definition). Additional motivating examples for this definition are given in [7].

The following proposition, which calculates the weak discrepancy of posets of the form $\mathbf{r}+\mathbf{s}$ appears implicitly in [8] and explicitly in [7].

Proposition $2 w d(\mathbf{r}+\mathbf{s})=\lceil(r+s-2) / 2\rceil$. 


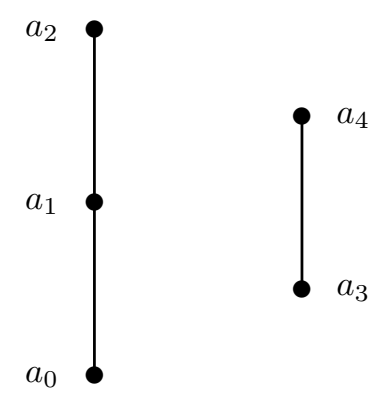

Figure 1: The poset $P=\mathbf{3}+\mathbf{2}$ with $w d(P)=2$ and $w d_{F}(P)=3 / 2$.

By Definition 1 the weak discrepancy of a poset is a nonnegative integer, and Proposition 2 demonstrates that each nonnegative integer is achieved as the weak discrepancy of a poset of the form $\mathbf{r}+\mathbf{s}$. Thus

$$
\{w d(P): P \text { a poset }\}=\{0,1,2,3, \ldots\} .
$$

As discussed above, linear orders (or chains) are posets with no induced $\mathbf{1}+\mathbf{1}$, that is no induced posets of the form $\mathbf{r}+\mathbf{s}$ where $r+s=2$. Weak orders are posets with no induced $\mathbf{2}+\mathbf{1}$, that is no induced posets of the form $\mathbf{r}+\mathbf{s}$ where $r+s=3$. The next natural class to consider is that of posets with no induced $\mathbf{r}+\mathbf{s}$ where $r+s=4$, that is posets with no induced $\mathbf{2}+\mathbf{2}$ and no induced $3+\mathbf{1}$. These are known as semiorders. By a theorem of Scott and Suppes [5], this class is equivalent to the class of unit interval orders, that is, posets which can be represented as follows: each element $x$ of the ground set is assigned a unit length interval $I_{x}$ on the real number line so that $x \prec y$ if and only if the interval $I_{x}$ is completely to the left of $I_{y}$. As a consequence of these definitions, $\{$ linear orders $\} \subset\{$ weak orders $\} \subset\{$ semiorders $\}$.

As discussed above, the weak discrepancy of a linear order or a weak order is 0 . The weak discrepancy of a semiorder is calculated in [7] as follows:

Proposition 3 If $P$ is a semiorder then $w d(P) \leq 1$. In particular,

$$
w d(P)= \begin{cases}1, & \text { if } P \text { has an induced } \mathbf{2}+\mathbf{1} \text { (semiorder but not weak) } \\ 0, & \text { otherwise (weak order). }\end{cases}
$$

An immediate consequence of Proposition 3 is

$$
\{w d(P): P \text { a semiorder }\}=\{0,1\} .
$$

In this paper we prove results analogous to equations (1) and (2) for fractional weak discrepancy, and construct examples to show how each value is realized. 


\subsection{Fractional Weak Discrepancy}

In [6] we express the weak discrepancy problem as an integer program. The linear relaxation of this integer program gives a fractional version, which can be interpreted as a variant of the salary assignment problem described in Section 1.1. Here we can think of $f(a)$ as the actual salary assigned to employee $a$, rather than as a salary level as in the definition of weak discrepancy. If we choose the units of $f(a)$ (dollars, hundreds of dollars, etc.) to make 1 unit a significant salary difference, then the values of $f(a)$ need not be integers.

Definition 4 The fractional weak discrepancy $w d_{F}(P)$ of a poset $P=(V, \prec)$ is the minimum nonnegative real number $k$ for which there exists a function $f: V \rightarrow \mathbf{R}$ satisfying

(i) if $a \prec b$ then $f(a)+1 \leq f(b) \quad$ ("up" constraints)

(ii) if $a \| b$ then $|f(a)-f(b)| \leq k$. ("side" constraints) Such a function $f$ is called an optimal fractional weak labeling of $P$ (or of $V$ ).

The poset $\mathbf{3}+\mathbf{2}$ shown in Figure 1 has fractional weak discrepancy equal to $3 / 2$. An optimal fractional weak labeling is given by $f\left(a_{0}\right)=0, f\left(a_{1}\right)=1$, $f\left(a_{2}\right)=2, f\left(a_{3}\right)=1 / 2, f\left(a_{4}\right)=3 / 2$.

Remark 5 In [6] we show that $w d_{F}(P)$ exists as the optimum value of a linear program, and so the minimum in Definition 4 is well defined. It follows from Definitions 1 and 4 that $w d_{F}(P) \leq w d(P)$ for all posets $P$.

By Definition 4, the fractional weak discrepancy is a nonnegative real number. To show that it is always a rational number, we need the characterization of fractional weak discrepancy using the notion of forcing cycles.

Definition 6 A forcing cycle $C$ of poset $P=(V, \prec)$ is a sequence $C: a_{0}, a_{1}, \ldots, a_{m}=$ $a_{0}$ of $m \geq 2$ elements of $V$ for which $a_{i} \prec a_{i+1}$ or $a_{i} \| a_{i+1}$ for each $i: 0 \leq i<m$. If $C$ is a forcing cycle, we write $u p(C)=\left|\left\{i: a_{i} \prec a_{i+1}\right\}\right|$ and $\operatorname{side}(C)=\mid\{i$ : $\left.a_{i} \| a_{i+1}\right\} \mid$.

In [2], forcing cycles are called picycles (preference-indifference cycles). Note that if $P$ has no incomparable pair then it is a linear order, has no forcing cycle, and $w d_{F}(P)=0$. The following result characterizes fractional weak discrepancy in terms of forcing cycles when $P$ has an incomparable pair. The analogous result for weak discrepancy appears in [3].

Theorem $7([\mathbf{6}])$ Let $P=(V, \prec)$ be a poset with at least one incomparable pair. Then $w d_{F}(P)=\max _{C} \frac{u p(C)}{\text { side }(C)}$, taken over all forcing cycles $C$ in $P$.

By Definition 4 and Theorem $7, w d_{F}(P)$ is nonnegative and rational. However, as the following shows, not every nonnegative rational number can be achieved as the fractional weak discrepancy of a poset.

Corollary 8 The fractional weak discrepancy of any poset is a nonnegative rational number that cannot take any value strictly between 0 and $1 / 2$. 
Proof. If a poset $P$ does not contain an induced $\mathbf{2}+\mathbf{1}$ then it is a weak order and $w d_{F}(P)=0$. If it does contain $\mathbf{2}+\mathbf{1}$ (with elements $a \prec b$, and $c$ ) then it contains the forcing cycle $C: a \prec b\|c\| a$ with $u p(C)=1$ and $\operatorname{side}(C)=2$, and thus by Theorem $7, w d_{F}(P) \geq 1 / 2$.

For the poset in Figure 1, the maximum ratio is achieved for the forcing cycle $C: a_{0} \prec a_{1} \prec a_{2}\left\|a_{3} \prec a_{4}\right\| a_{5}=a_{0}$ with $u p(C)=3$ and side $(C)=2$. Notice that for the optimal fractional labeling $f$ given earlier, $f\left(a_{i+1}\right)=f\left(a_{i}\right)+1$ whenever $a_{i} \prec a_{i+1}$ and $f\left(a_{i+1}\right)=f\left(a_{i}\right)-3 / 2$ whenever $a_{i} \| a_{i+1}$. The next proposition shows that these equalities hold in general.

Proposition 9 Let $P=(V, \prec)$ be a poset with $w d_{F}(P)=k$. Let $C: a_{0}, a_{1}, a_{2}, \ldots, a_{m}=$ $a_{0}$ be a forcing cycle with $\frac{u p(C)}{\operatorname{side}(C)}=k$ and let $f: V \rightarrow \mathbf{R}$ be a function that satisfies (i) and (ii) of Definition 4 for this optimal value of $k$. Then $f\left(a_{i+1}\right)=f\left(a_{i}\right)+1$ whenever $a_{i} \prec a_{i+1}$ and $f\left(a_{i+1}\right)=f\left(a_{i}\right)-k$ whenever $a_{i} \| a_{i+1}$.

Proof. By Definition 4, for each $i$ with $a_{i} \prec a_{i+1}$ we have $f\left(a_{i+1}\right) \geq f\left(a_{i}\right)+1$ and for each $i$ with $a_{i} \| a_{i+1}$ we have $f\left(a_{i+1}\right) \geq f\left(a_{i}\right)-k$. Summing over all $i$ we get

$0=\sum_{i=0}^{m-1} f\left(a_{i+1}\right)-f\left(a_{i}\right) \geq u p(C)-k \cdot \operatorname{side}(C)=u p(C)-\frac{u p(C)}{\operatorname{side}(C)} \operatorname{side}(C)=0$.

So equality must hold for each term, that is, $f\left(a_{i+1}\right)=f\left(a_{i}\right)+1$ whenever $a_{i} \prec a_{i+1}$ and $f\left(a_{i+1}\right)=f\left(a_{i}\right)-k$ whenever $a_{i} \| a_{i+1}$.

Proposition 9 can also be proved using the linear programming duality framework of $[6]$ and the principle of linear complementarity.

\section{Fractional Weak Discrepancy of Semiorders}

In this section we prove that the fractional weak discrepancy of a poset $P$ is less than one if and only if $P$ is a semiorder. As discussed in the proof of Corollary 8 , posets that are not weak orders (i.e., those containing $\mathbf{2}+\mathbf{1}$ ) have weak discrepancy strictly greater than 0 . The following proof is similar.

Proposition 10 If $w d_{F}(P)<1$ then $P$ is a semiorder.

Proof. If $P$ is not a semiorder then $P$ contains either a $\mathbf{2}+\mathbf{2}$ (with elements $a \prec b$ and $c \prec d$ ) or a $\mathbf{3}+\mathbf{1}$ (with elements $x \prec y \prec z$ and $w$ ). The former contains a forcing cycle $C: a \prec b\|c \prec d\| a$ and the latter contains a forcing cycle $C^{\prime}: x \prec y \prec z\|w\| x$ with $u p(C)=u p\left(C^{\prime}\right)=2$ and $\operatorname{side}(C)=$ $\operatorname{side}\left(C^{\prime}\right)=2$. Thus by Theorem $7, w d_{F}(P) \geq 2 / 2=1$.

We know from Proposition 3 that the weak discrepancy of a semiorder $P$ is either 0 (weak order) or 1 (not a weak order). In addition, $0 \leq w d_{F}(P) \leq w d(P)$ 
by Remark 5. Thus semiorders $P$ with $w d(P)=0$ also have $w d_{F}(P)=0$, and those with $w d(P)=1$ will have $1 / 2 \leq w d_{F}(P) \leq 1$ by Corollary 8. In Theorem 18 we will show exactly which values are achieved as the fractional weak discrepancy of a semiorder. The following result shows that 1 is never achieved, that is, $w_{F}(P)<1$. Thus the only semiorders $P$ with $w d_{F}(P)=$ $w d(P)$ are the weak orders.

Recall from the introduction that the class of semiorders is equivalent to the class of unit interval orders. Thus a semiorder $P=(V, \prec)$ has a representation by a set of intervals in the real line $\left\{I_{x} \mid x \in V\right\}$ so that $x \prec y$ if and only if $I_{x}$ is completely to the left of $I_{y}$. Indeed, it is always possible to find such a representation in which all endpoints of intervals are distinct (e.g., see [4]). For convenience we write $I_{x}=[L(x), R(x)]$. We require the following definition.

Definition 11 A fractional $k$-weak labeling of $P$ is a function $f$ on $V$ that satisfies conditions (i) and (ii) of Definition 4 for some $k \geq 0$.

Proposition 12 Let $P=(V, \prec)$ be a semiorder. Then $w d_{F}(P)<1$. Moreover, for any unit interval representation of $P$ with distinct endpoints, $\left\{I_{x} \mid x \in V\right\}$, there exist both a number $k<1$ and a fractional $k$-weak labeling $f$ of $P$ that is strictly increasing on $\{L(x): x \in V\}$, i.e.,

$$
\text { for all } x, y \in V \text { if } L(x)<L(y) \text { then } f(x)<f(y) \text {. }
$$

Proof. The proof proceeds by induction on $|V|$. The base case $|V|=1$ is trivial, since then $w d_{F}(P)=0$ and (3) is satisfied vacuously by any labeling.

Now suppose the result is true for all semiorders with up to $n$ elements and let $P=(V, \prec)$ be a semiorder with $|V|=n+1$. Let $\left\{I_{x} \mid x \in V\right\}$ be a unit interval representation of $P$ with distinct endpoints and let $I_{r}$ be the interval with the largest left endpoint, $L(r)=\max _{x \in V} L(x)$. Define $W=V \backslash\{r\}$ and let $Q=(W, \prec)$ be the corresponding induced poset. Certainly $\left\{I_{x} \mid x \in W\right\}$ is a unit interval representation of $Q$, so $Q$ is a semiorder. Since $|W|=n$, the induction hypothesis implies that $w d_{F}(Q)<1$ and there exists some $k<1$ and a fractional $k$-weak labeling $f$ satisfying (3) on $W$.

Let $\epsilon=1-k$. Since $0 \leq k<1$ we have $0<\epsilon \leq 1$. We extend the labeling $f$ to $V$ by defining $f(r)$ as follows. If $\{x \in W: x \prec r\} \neq \emptyset$, then there exists some $a \in W$ with $a \prec r$ and $L(a)=\max _{x \prec r} L(x)$. If $\{x \in W: x \| r\} \neq \emptyset$, then there exists some $b \in W$ with $b \| r$ and $L(b)=\max _{x \| r} L(x)$. By definition of $r$ and since $|V| \geq 2$, at least one of $a, b$ exists.

If $x \prec r$ for all $x \in W$ then $a$ exists but $b$ does not. In this case we define $f(r)=f(a)+1$. It is easy to show that $w d_{F}(P)=w d_{F}(Q)<1$ and that, for the same value of $k$ as on $W, f$ is a fractional $k$-weak labeling of $P$ satisfying (3) on $V$.

If every element of $W$ is incomparable to $r$, then $b$ exists but $a$ does not. In this case we define $f(r)=f(b)+\frac{\epsilon}{2}$. Again, it is easy to show $f$ is a fractional $\left(k+\frac{\epsilon}{2}\right)$-weak labeling of $P, w d_{F}(P) \leq k+\frac{\epsilon}{2}<1$, and $f$ satisfies (3) on $V$.

Otherwise, both $a$ and $b$ exist. Now we define

$$
f(r)=\max \left\{f(a)+1, f(b)+\frac{\epsilon}{2}\right\} .
$$


We begin by proving that $f$ satisfies (3) on $V$. Let $x, y \in V$ and let $L(x)<$ $L(y)$. In particular, $x \neq r$. If $y \neq r$ then (3) holds by the induction hypothesis. Suppose $y=r$. If $x \prec r$ then $L(x) \leq L(a)$ by definition of $a$, so $f(x) \leq f(a)<$ $f(r)=f(y)$ by the induction hypothesis and the definition of $f(r)$. Similarly, if $x \| r$ then $L(x) \leq L(b)$ so $f(x) \leq f(b)<f(r)=f(y)$. By the choice of $r$, we know $r \prec x$ is impossible. Thus in all cases, (3) holds on $V$.

Next we show that the extended function $f$ satisfies the up and side constraints of Definition 4 for some $h, k \leq h<1$. Since $f$ satisfies them for $Q$, we need only show the constraints hold for $x, r$ where $x \in W$.

To verify the up constraints note that, as above, $r \prec x$ is impossible. Suppose $x \prec r$. By (3), our choice of $a$, and the fact that the representing intervals have distinct endpoints, $f(x) \leq f(a)$. Thus, $f(x)+1 \leq f(a)+1 \leq f(r)$, as required.

To verify the side constraints, let $x|| r$. We will show $|f(x)-f(r)|<1$. Since $a \prec r$ and the representing intervals all have the same length, $L(a)<L(x)$. By (3), $f(a)<f(x)$. There are two cases to consider depending on whether (I) $f(r)=f(a)+1$ or (II) $f(r)=f(b)+\frac{\epsilon}{2}$.

Suppose (I) holds. By definition of $r$ and since the representing intervals have distinct endpoints, $L(x)<L(r)$. By $(3), f(a)<f(x)<f(r)$ and thus

$$
0<f(r)-f(x)<f(r)-f(a)=1,
$$

as required.

Suppose (II) holds. Since $r$ is incomparable to both $b$ and $x$, and since $L(r)$ is maximum among all left endpoints of the unit interval representation of $P$, $L(r) \in I_{x} \cap I_{b} \neq \emptyset$ and so $x \| b$. Since $f$ is a fractional $k$-weak labeling of $Q$ and by definition of $b$,

$$
0 \leq f(b)-f(x) \leq k=1-\epsilon
$$

But $f(r)=f(b)+\frac{\epsilon}{2}$, so

$$
0 \leq\left(f(r)-\frac{\epsilon}{2}\right)-f(x) \leq 1-\epsilon
$$

and hence

$$
\frac{\epsilon}{2} \leq f(r)-f(x) \leq 1-\frac{\epsilon}{2}<1
$$

as required.

Thus $|f(x)-f(r)|<1$ for all $x|| r$. Since $V$ is finite, there is some $h<1$ for which $f$ is a fractional $h$-weak labeling of $P$. Since the fractional weak discrepancy of $P$ is the minimum such $h, w d_{F}(P) \leq h<1$.

Propositions 10 and 12 immediately yield the following.

Corollary $13 A$ poset $P$ is a semiorder if and only if $w d_{F}(P)<1$.

\section{Range of $w d_{F}$ for Non-semiorders}

In this section we describe the values that can be taken by $w d_{F}(P)$ for posets $P$ that are not semiorders. Proposition 10 implies that these values must be at 
least one. The next proposition shows we can achieve any rational number that is at least one as the fractional weak discrepancy of some poset. Our proof is constructive.

Proposition 14 If $q \in \mathbf{Q}$ and $q \geq 1$ then there exists a partial order with fractional weak discrepancy equal to $q$.

Proof. Let $q \geq 1$ be a rational number. We will construct a poset $P=(V, \prec)$ with $w d_{F}(P)=q$. Write $q=\frac{m}{n}$, not necessarily in lowest terms, for integers $m \geq n>1$ (if $q$ is an integer we can write $q=\frac{2 q}{2}$ ). First we will show that $w d_{F}(P) \leq \frac{m}{n}$ by exhibiting a labeling function $f$ satisfying properties (i), (ii) of Definition 4 with $k=\frac{m}{n}$. To simplify the argument, we will first construct a labeling function $g: V \rightarrow \mathbf{R}$ satisfying the equivalent conditions

$$
\begin{aligned}
& \text { (i) } \quad \text { if } u \prec v \text { then } g(v) \geq g(u)+n \\
& \text { (ii) } \quad \text { if } u|| v \text { then }|g(u)-g(v)| \leq m
\end{aligned}
$$

and then set $f(u)=\frac{g(u)}{n}$.

We start with a poset $P^{\prime}=\left(V, \prec^{\prime}\right)=\mathbf{2}+\cdots+\mathbf{2}+(\mathbf{m}-\mathbf{n}+\mathbf{2})$ consisting of $n-1$ chains of height 2 and one chain of height $m-n+2$ :

$$
\begin{gathered}
x_{1} \prec^{\prime} y_{1}, x_{2} \prec^{\prime} y_{2}, \ldots, x_{n-1} \prec^{\prime} y_{n-1} \\
z_{1} \prec^{\prime} z_{2} \prec^{\prime} \ldots \prec^{\prime} z_{m-n+2} .
\end{gathered}
$$

There are no additional comparabilities in $P^{\prime}$ beyond those implied by transitivity. The poset $P^{\prime}$ contains the forcing cycle $C$ :

$$
x_{1} \prec^{\prime} y_{1}\left\|^{\prime} x_{2} \prec^{\prime} y_{2}\right\|^{\prime} \ldots\left\|^{\prime} x_{n-1} \prec^{\prime} y_{n-1}\right\|^{\prime} z_{1} \prec^{\prime} z_{2} \prec^{\prime} \ldots \prec^{\prime} z_{m-n+2} \|^{\prime} x_{1}
$$

with $u p(C)=(n-1)+(m-n+1)=m$ and $\operatorname{side}(C)=n$. (Note that if we were to permit $n=1$ when $q$ is an integer then $P^{\prime}$ would consist of a single chain and have no side arcs or forcing cycle.)

We next label the elements of $V$ so that consecutive elements of $C$ satisfy

$$
\begin{array}{ll}
\left(\mathrm{i}^{\prime}\right) & \text { if } u \prec^{\prime} v \text { then } g(v)=g(u)+n \\
\left(\mathrm{ii}^{\prime}\right) & \text { if } u \|^{\prime} v \text { then } g(v)=g(u)-m .
\end{array}
$$

The following is an explicit labeling satisfying conditions (5):

$$
\begin{array}{llll}
g\left(x_{1}\right) & =0 & g\left(y_{1}\right) & =n \\
g\left(x_{2}\right) & =n-m & g\left(y_{2}\right) & =2 n-m \\
& \vdots & & \vdots \\
g\left(x_{n-1}\right) & =(n-2)(n-m) & g\left(y_{n-1}\right) & =(n-2)(n-m)+n
\end{array}
$$

and

$$
g\left(z_{r}\right)=(n-1)(n-m)+(r-1) n \text { for } r=1,2, \ldots, m-n+2 .
$$


The labeling function $g$ satisfies condition (i) of our goal (4) because for all $u, v \in V$ with $u \prec^{\prime} v$ (including those precedences that follow from transitivity), we have $g(v) \geq g(u)+n$. However, $g$ may not satisfy condition (ii) of (4) so we add the following comparabilities to those in $P^{\prime}$. If $\left.u\right|^{\prime} v$ and $|g(u)-g(v)|>m$, then we add the comparability

$$
\begin{cases}u \prec v & \text { if } g(v)>g(u)+m \\ v \prec u & \text { if } g(v)<g(u)-m .\end{cases}
$$

Let $P=(V, \prec)$, where $\prec$ is the relation on $V$ that includes the comparabilities in $P^{\prime}$ together with those added in (6). We must show that $P$ is a partial order and that $w d_{F}(P)=\frac{m}{n}$.

Since $|g(u)-g(u)|=0<m$ we did not add any comparabilities of the form $u \prec u$ and so $P$ is irreflexive. We show that $P$ is transitive and antisymmetry follows as a consequence. Suppose $u \prec v$ and $v \prec w$. We show $u \prec w$, dividing the argument into four cases according to how $u, v, w$ are related in $P^{\prime}=\left(V, \prec^{\prime}\right)$.

Case 1. $u \prec^{\prime} v$ and $v \prec^{\prime} w$ : Then $u \prec^{\prime} w$ by transitivity in $P^{\prime}$, so $u \prec w$.

Case 2. $u \prec^{\prime} v$ and $v \|^{\prime} w$ : In this case, $v \prec w$ was added in (6). Thus,

$$
\begin{array}{lll}
u \prec^{\prime} v & \Rightarrow & g(v) \geq g(u)+n \\
v \|^{\prime} w, v \prec w & \Rightarrow \quad g(v)<g(w)-m .
\end{array}
$$

Combining these two yields

$$
g(u) \leq g(v)-n<g(w)-m-n<g(w)-m .
$$

If $u \|^{\prime} w$ then applying first (7) and then (6) gives $u \prec w$, as desired. If $u \prec^{\prime} w$ then we also have $u \prec w$. Finally, if $w \prec^{\prime} u$ then $w \prec^{\prime} u \prec^{\prime} v$, a contradiction. So in the only possible subcases, we have $u \prec w$.

Case 3. $u \|^{\prime} v$ and $v \prec^{\prime} w$ : This is similar to Case 2 .

Case 4. $u \|^{\prime} v$ and $v \|^{\prime} w$ : In this case, both $u \prec v$ and $v \prec w$ were added in (6). Thus,

$$
\begin{aligned}
& u \|^{\prime} v, u \prec v \quad \Rightarrow \quad g(u)<g(v)-m \\
& v \|^{\prime} w, v \prec w \quad \Rightarrow \quad g(v)<g(w)-m .
\end{aligned}
$$

Hence,

$$
g(u)<g(v)-m<g(w)-2 m<g(w)-m .
$$

If $u \prec^{\prime} w$ in $P^{\prime}$ then $u \prec w$. If $w \prec^{\prime} u$ then $g(u) \geq g(w)+n>g(w)$, contradicting (8). If $u \|^{\prime} w$ then (8) and (6) imply $u \prec w$. So again in the only possible subcases, $u \prec w$.

Thus $\prec$ is transitive and so $P=(V, \prec)$ is a partial order. The labeling function $g$ achieves (ii) of our goal conditions (4) for the poset $P$ because we have eliminated the incomparabilities for which (ii) was violated. We have 
already shown that it achieves (i) of (4) for the original comparabilities in $P^{\prime}$. It also achieves (i) of (4) for the comparabilities we added, because if $u \prec v$ and $u \|^{\prime} v$ then $g(v)>g(u)+m \geq g(u)+n$, since $q=\frac{m}{n} \geq 1$. Thus $f(u)=\frac{g(u)}{n}$ is a $q$-weak labeling of $P$ and $w d_{F}(P) \leq q$, by Definition 4 .

Finally note that by (5), no additional comparabilities were added between consecutive elements of the cycle $C$. Thus, $C$ remains a forcing cycle in $P$. Since, $u p(C)=m$ and side $(C)=n$, Theorem 7 implies that $w d_{F}(P) \geq \frac{m}{n}=q$. This proves $w d_{F}(P)=q$.

The main result of this section now follows immediately from Corollaries 8 and 13 and Proposition 14.

Theorem 15 If $P$ is a poset that is not a semiorder then $w d_{F}(P)$ is a rational number that is at least one. Furthermore, for each rational number $q \geq 1$, there exists a poset $P$ (that is not a semiorder) with $w d_{F}(P)=q$. Equivalently, $\left\{w d_{F}(P): P\right.$ a poset that is not a semiorder $\}=\{q \geq 1: q \in \mathbf{Q}\}$.

\section{$4 \quad$ Range of $w d_{F}$ for Semiorders}

Recall from Corollary 13 that $P$ is a semiorder if and only if $w d_{F}(P)<1$. In this section we characterize those values less than one that can be achieved as the fractional weak discrepancy of a semiorder. We begin with a construction that shows, for each $r \geq 0$, how to achieve $\frac{r}{r+1}$ as $w d_{F}(P)$ for some semiorder $P$.

Proposition 16 For each integer $r \geq 0$ there exists a semiorder $P$ with $w d_{F}(P)=$ $\frac{r}{r+1}$.

Proof. For $r=0$, let $P$ be any linear order; then $P$ is a semiorder with $w d_{F}(P)=0$. Now let $r$ be an integer with $r \geq 1$. We will construct a semiorder $P$ by constructing a unit interval representation, where all intervals have the same length and $x \prec y$ in $P$ if and only if the interval corresponding to $x$ lies entirely to the left of the one corresponding to $y$.

We define intervals $I_{j}=\left[\alpha_{j}, \beta_{j}\right]$ for $j=1, \ldots, r+1$ and $I_{j}^{\prime}=\left[\alpha_{j}^{\prime}, \beta_{j}^{\prime}\right]$ for $j=1, \ldots, r$ recursively as follows. Let

(1) $\alpha_{1}=0$

$\left(1^{\prime}\right) \alpha_{1}^{\prime}=2 r$

(2) $\beta_{j}=\alpha_{j}+2 r+1, \alpha_{j+1}=\beta_{j}+1$

$\left(2^{\prime}\right) \beta_{j}^{\prime}=\alpha_{j}^{\prime}+2 r+1, \alpha_{j+1}^{\prime}=\beta_{j}^{\prime}-1$.

These relations yield the following intervals, all with length $2 r+1$ :

$$
I_{j}=[2(j-1)(r+1), 2 j(r+1)-1], \quad I_{j}^{\prime}=[2 j r, 2(j+1) r+1] .
$$

Figure 2 illustrates the construction for $r=3$.

This unit interval representation defines a semiorder $P=\left\{x_{1}, \ldots, x_{r+1}, x_{1}^{\prime}, \ldots, x_{r}^{\prime}\right\}$, where the elements $x_{j}, x_{j}^{\prime}$ correspond to the intervals $I_{j}, I_{j}^{\prime}$, respectively. For 


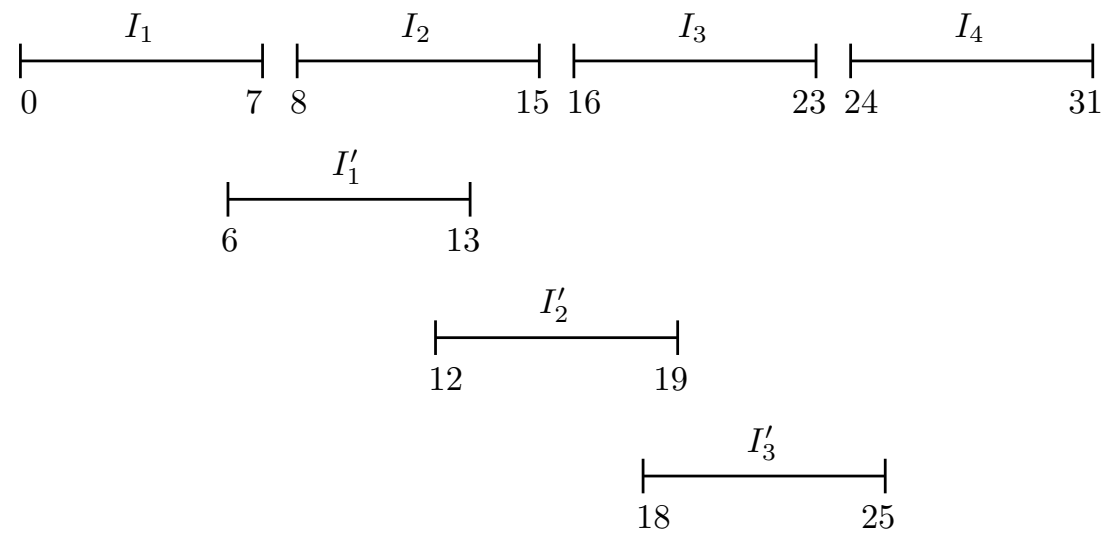

Figure 2: The intervals $I_{j}, I_{j}^{\prime}$ in the case $r=3$.

$j=1, \ldots, r$, the following properties (a) $-(\mathrm{g})$ together with transitivity describe all the precedence and incomparability relations in $P$. Properties (a), (b) follow immediately from the definitions of $I_{j}, I_{j}^{\prime}$. We will also verify (g).
(a) $x_{1} \prec x_{2} \prec \cdots \prec x_{r+1}$
(e) $x_{i}^{\prime} \prec x_{j}$ for $i=1, \ldots, j-2$
(b) $x_{1}^{\prime}\left\|x_{2}^{\prime}\right\| \cdots \| x_{r}^{\prime}$
(f) $x_{j} \prec x_{i}^{\prime}$ for all $i=j+1, \ldots r$
(c) $x_{j} \| x_{j}^{\prime}$
(d) $x_{j} \| x_{j-1}^{\prime}($ for $j \geq 2)$
(g) $x_{j}^{\prime} \prec x_{i}^{\prime}$ for all $i=j+2, \ldots r$.

To prove (g), let $j+2 \leq i \leq r$. Then

$$
\alpha_{i}^{\prime}-\beta_{j}^{\prime}=2 i r-(2(j+1) r+1)=2(i-(j+1)) r-1>2 r-1>0,
$$

so $x_{j}^{\prime} \prec x_{i}^{\prime}$. The remaining properties follow similarly.

By (a)-(d), the sequence

$$
C: x_{1} \prec x_{2} \prec \cdots \prec x_{r+1}\left\|x_{r}^{\prime}\right\| x_{r-1}^{\prime}\|\cdots\| x_{1}^{\prime} \| x_{1}
$$

is a forcing cycle in $P$. Since $u p(C)=r$ and $\operatorname{side}(C)=r+1$, we obtain the lower bound $w d_{F}(P) \geq \frac{r}{r+1}$.

We next find an upper bound for $w d_{F}(P)$ by labeling the elements of $P$. Let

$$
f\left(x_{j}\right)=j, \quad f\left(x_{j}^{\prime}\right)=1+j\left(\frac{r}{r+1}\right) .
$$

We must now check that $f$ satisfies the up and side constraints of Definition 4 for cases (a)-(g). We will verify them for cases (a), (b), (c), and (e).

The up constraints corresponding to the precedence relations (a) follow directly from (9). Similarly, the side constraints for the incomparability relations 
in (b) are satisfied for $k=\frac{r}{r+1}$, by (9). We prove they are also satisfied for those in (c) using this value of $k$. For $1 \leq j \leq r$,

$$
f\left(x_{j}^{\prime}\right)-f\left(x_{j}\right)=1+j\left(\frac{r}{r+1}\right)-j=1-\frac{j}{r+1},
$$

so

$$
\frac{1}{r+1} \leq f\left(x_{j}^{\prime}\right)-f\left(x_{j}\right) \leq \frac{r}{r+1} .
$$

Finally, we check the up constraints in (e). For $1 \leq i \leq j-2$,

$f\left(x_{j}\right)-f\left(x_{i}^{\prime}\right)=j-\left(1+i\left(\frac{r}{r+1}\right)\right) \geq i+1-i\left(\frac{r}{r+1}\right) \geq i\left(1-\frac{r}{r+1}\right)+1>1$.

The remaining cases are checked similarly. This labeling gives the upper bound $w d_{F}(P) \leq \frac{r}{r+1}$. Therefore, $w d_{F}(P)=\frac{r}{r+1}$.

Next we show that any poset $P$ with $w d_{F}(P)<1$ has $w d_{F}(P) \in\left\{0, \frac{1}{2}, \frac{2}{3}, \frac{3}{4}, \frac{4}{5}, \ldots\right\}$. Thus we can never achieve numbers like $2 / 5$ or $7 / 9$ as the fractional weak discrepancy of a poset.

Proposition 17 If $P$ is a partial order with $w d_{F}(P)<1$, then $w d_{F}(P)=\frac{r}{r+1}$ for some nonnegative integer $r$.

Proof. Let $P$ be a poset with $w d_{F}(P)<1$. If $w d_{F}(P)=0$, then the conclusion follows with $r=0$. Otherwise, by Corollary $8, w d_{F}(P)=\frac{a}{b}$ for integers $a, b$. We may assume this fraction is in lowest terms and $a, b>0$; thus $\operatorname{gcd}(a, b)=1$, and since $w d_{F}(P)<1$ we have $a<b$. If $a=b-1$, we are done with $r=a$. Now we assume that $a<b-1$ and derive a contradiction. By Theorem 7 there exists a forcing cycle $C: x_{0}, x_{1}, \ldots, x_{m-1}=x_{0}$ with $\frac{u p(C)}{\operatorname{side}(C)}=w d_{F}(P)=\frac{a}{b}$. Then $m$ is a multiple of $a+b$.

Let $f: V \rightarrow \mathbf{R}$ be a labeling of $P$ that satisfies the two conditions of Definition 4 with $k=a / b$. Without loss of generality, suppose $f\left(x_{0}\right)=0$. By Proposition 9, $f\left(x_{i+1}\right)=f\left(x_{i}\right)+1$ whenever $x_{i} \prec x_{i+1}$ and $f\left(x_{i+1}\right)=f\left(x_{i}\right)-\frac{a}{b}$ whenever $x_{i} \| x_{i+1}$. Equivalently, the function $g(x)=b f(x)$ satisfies

$$
g\left(x_{i+1}\right)= \begin{cases}g\left(x_{i}\right)+b & \text { if } x_{i} \prec x_{i+1} \\ g\left(x_{i}\right)-a & \text { if } x_{i} \| x_{i+1}\end{cases}
$$

Since $a<b-1$, there exists an integer $c$ such that $a<c<b$. We seek a contradiction by producing a pair of elements $x, y \in V$ with $g(y)-g(x)=c$. For given such a pair, if $x \prec y$ then

$$
f(y)-f(x)=\frac{g(y)-g(x)}{b}=\frac{c}{b}<1,
$$

contradicting (i) of Definition 4. If $y \prec x$ then $f(x)-f(y)=-\frac{c}{b}<0$, which also contradicts (i). If $x \| y$ then

$$
|f(x)-f(y)|=\frac{c}{b}>\frac{a}{b},
$$


which contradicts (ii) of Definition 4 since $w d_{F}(P)=\frac{a}{b}$.

Equation (10) together with the fact that $g\left(x_{0}\right)=b f\left(x_{0}\right)=0$ and $b \equiv$ $-a(\bmod a+b)$ implies that

$$
g\left(x_{i}\right) \equiv i b(\bmod a+b)
$$

for $0 \leq i \leq m-1$. Since $\operatorname{gcd}(a, b)=1$, we know $\operatorname{gcd}(b, a+b)=1$ and thus $b$ has an inverse $\bmod (a+b)$. So $b k \equiv c(\bmod a+b)$ has a unique solution $k$ with $0<k<a+b$.

Define $\Delta g_{i, k}=g\left(x_{i+k}\right)-g\left(x_{i}\right)$ where all subscripts are taken mod $m$, the number of terms in the forcing cycle. Observe that

$$
\sum_{i=0}^{m-1} \Delta g_{i, k}=0
$$

since each $g\left(x_{i}\right)$ appears exactly once with a positive sign and exactly once with a negative sign. Furthermore, since subscripts are taken $\bmod m$ and $m$ is a multiple of $a+b,(11)$ remains valid for $i \geq m$. Thus for all $i \geq 0$,

$$
\begin{aligned}
\Delta g_{i, k} & =g\left(x_{i+k}\right)-g\left(x_{i}\right) \\
& \equiv(i+k) b-i b(\bmod a+b) \\
& \equiv k b(\bmod a+b) \\
& \equiv c(\bmod a+b),
\end{aligned}
$$

by the definition of $k$. By (10),

$$
\begin{aligned}
\Delta g_{i+1, k} & =g\left(x_{i+k+1}\right)-g\left(x_{i+1}\right) \\
& =g\left(x_{i+k}\right)\left\{\begin{array}{c}
+b \\
-a
\end{array}\right\}-g\left(x_{i}\right)\left\{\begin{array}{c}
+b \\
-a
\end{array}\right\} \\
& =g\left(x_{i+k}\right)-g\left(x_{i}\right)+\left\{\begin{array}{c}
0 \\
a+b \\
-(a+b)
\end{array}\right\} \\
& =\Delta g_{i, k}+\left\{\begin{array}{c}
0 \\
a+b \\
-(a+b)
\end{array}\right\} .
\end{aligned}
$$

Recall that our goal is to find $x, y \in V$ with $g(y)-g(x)=c$. If $\Delta g_{0, k}=$ $g\left(x_{k}\right)-g\left(x_{0}\right)=c$, we are done by setting $y=x_{k}, x=x_{0}$. Suppose now that $\Delta g_{0, k}>c$. Since $\sum_{i=0}^{m-1} \Delta g_{i, k}=0$, there must be some $h$ such that $\Delta g_{h, k}>0$ and $\Delta g_{h+1, k}<0$. We will prove that $\Delta g_{h, k}=c$. If $\Delta g_{h, k}>c$, then (12) implies $\Delta g_{h, k} \geq c+(a+b)$. So by (13),

$$
\begin{aligned}
\Delta g_{h+1, k} & =\Delta g_{h, k}-(a+b) \\
& \geq c+(a+b)-(a+b) \\
& =c>0
\end{aligned}
$$


contradicting the fact that $\Delta g_{h+1, k}<0$. A similar argument may be given when $\Delta g_{0, k}<c$. We conclude that $\Delta g_{h, k}=c$, and so we are done by setting $y=x_{h+k}, x=x_{h}$. 17.

The main result of this section now follows from Propositions 12, 16, and

Theorem 18 If $P$ is a semiorder then $w d_{F}(P)=\frac{r}{r+1}$ for some integer $r \geq 0$. Furthermore, for each integer $r \geq 0$, there exists a semiorder $P$ with $w d_{F}(P)=$ $\frac{r}{r+1}$. Equivalently, $\left\{w d_{F}(P): P\right.$ a semiorder $\}=\left\{0, \frac{1}{2}, \frac{2}{3}, \frac{3}{4}, \frac{4}{5}, \ldots\right\}$.

Combining Theorems 15 and 18 yields the following result that characterizes those values that can be achieved as the fractional weak discrepancy of a poset.

Corollary $19\left\{w d_{F}(P): P\right.$ a poset $\}=\{q \geq 1: q \in \mathbf{Q}\} \cup\left\{0, \frac{1}{2}, \frac{2}{3}, \frac{3}{4}, \frac{4}{5}, \ldots\right\}$.

\section{References}

[1] K.P. Bogart. Introductory Combinatorics. Harcourt Brace Jovanovich, New York, 1990.

[2] P.C. Fishburn. Interval orders and interval graphs: A study of partially ordered sets. John Wiley \& Sons, New York, 1985.

[3] J.G. Gimbel and A.N. Trenk. On the weakness of an ordered set. SIAM J. Discrete Math., 11:655-663, 1998.

[4] M.C. Golumbic and A.N. Trenk. Tolerance Graphs. Cambridge University Press, Cambridge, 2004.

[5] D. Scott and P. Suppes. Foundational aspects of theories of measurement. J. Symbolic Logic, 23:113-128, 1958.

[6] A. Shuchat, R. Shull, and A. Trenk. The fractional weak discrepancy of a partially ordered set. Submitted.

[7] P.J. Tanenbaum, A.N. Trenk, and P.C. Fishburn. Linear discrepancy and weak discrepancy of partially ordered sets. ORDER, 18:201-225, 2001.

[8] A.N. Trenk. On $k$-weak orders: Recognition and a tolerance result. Discrete Math., 181:223-237, 1998. 